\title{
Test de estimulación intraarterial selectiva con calcio en el diagnóstico de localización de insulinomas. Casos clínicos
}

\author{
Darío Martínez $0^{1}$, G uillermo Silva $Q^{2}$, Iván Solís $0^{2}$, \\ Jorge Toloza $\mathrm{H}^{2}$, Carlos Benavides $\mathrm{C}^{3}$, James $\mathrm{H}$ amilton $\mathrm{S}^{4}$, \\ Fernando 0 sorio $\mathrm{G}^{4}$, G ermán Iñiguez $\mathrm{V}^{\mathrm{a}}$, Néstor Soto $\mathrm{I}^{2}$. \\ Selective intra arterial calcium \\ stimulation for the localization of \\ insulinomas. Report of two cases
} \begin{abstract}
tations are frequently confounded with neuropsychiatric symptoms, and definitive diagnosis can be delayed for a long time. These tumors are usually small, of less than $2 \mathrm{~cm}$. Thus, their preoperative localization is difficult. We report two patients with a clinical diagnosis of insulinoma, in whom the preoperative imaging study was negative. Both fulfilled diagnostic criteria, with high serum insulin levels in the presence of a blood glucose of less than $45 \mathrm{mg} / \mathrm{dl}$. The imaging study, including abdominal computed tomography and pancreatic endoscopic ultrasonography did not disclose the location of the tumor. A pancreatic angiography with selective stimulation with intra arterial calcium and venous sampling for insulin measurements, was performed in both patients. This test allowed the exact localization of the tumors and their successful excision. A review of other localization diagnostic tests is done (Rev Méd Chile 2004; 132: 71-5).
\end{abstract}

Insulinoma is the most common neuroendocrine tumor. Its clinical manifes-

(Key Words: Angiography; Insulinoma; Neuroendrocrine tumors)

Recibido el 22 de agosto, 2003. Aceptado el 21 de octubre, 2003.

Unidad de Hemodinamia ${ }^{1}$, Servicios de Medicina Interna ${ }^{2}$ y de Cirugía ${ }^{3}$, Hospital San BorjaArriarán. Servicios de Medicina Interna y de Cirugía, Hospital Padre Hurtado 4 .

aBioquímico, IDIMI, Universidad de Chile.

L os insulinomas son los tumores neuroendocrimos más frecuentes, pero a pesar de ello, presentan una baja incidencia, 0,5 a 4 casos por millón de habitantes al año ${ }^{1}$. En muchos casos su diagnóstico puede retrasarse meses 0 años. La variedad de síntomas neuroglucopénicos y adrenérgicos que caractenizan este tumor son confundidos con frecuencia con alteraciones psiquiátricas y cuadros comiciales.

Los criterios diagnósticos necesarios y suficientes para sospechar un insulinoma incluyen ${ }^{2}$ : hipoglice-

Correspondencia a: Dr. Néstor Soto. Santa Rosa 1234, $6^{\circ}$ piso, Santiago. Servicio de Medicina Interna.

Fono 5446600. Fax: 5546602. E mail: nestsoto@mi.cl mia espontánea inferior a $45 \mathrm{mg} / \mathrm{dL}$, insulinemia mayor a $6 \mu \mathrm{U} / \mathrm{mL}$, péptido $C$ mayor de $0,2 \mathrm{mmol} / \mathrm{L}$ tomados durante un episodio de hipoglicemia espontánea o durante un test de ayuno y negatividad en el rastreo de sulfonilureas en orina. Una vez confirmada la sospecha de insulinoma, se debe abordar la difícil tarea de localizar anatómicamente el tumor.

Sobre $80 \%$ de los insulinomas miden menos de $2 \mathrm{~cm}$ de diámetro, por lo cual su localización siempre ha sido difícil. La sensibilidad de métodos radiológicos convencionales como tomografía axial computada (TAC) abdominal y resonancia nuclear magnética no superan el $50 \%{ }^{3}$. La endosonografía 
tiene una sensibilidad aproximada al $80 \%$ en las mejores series ${ }^{4}$. El ultrasonido intraoperatorio permitiná, en un alto número de $\operatorname{casos}^{5}$ localizar el tumor, sugiriendo algunos que el análisis costobeneficio de un estudio preoperatorio de localización no sería favorable y con la exploración quirúrgica sería suficiente. La experiencia del cirujano es fundamental para el hallazgo, sin embargo, por la baja incidencia, es difícil acumular una gran experiencia quirúrgica. Nosotros creemos que la localización preoperatoria de un insulinoma es importante al momento de definir la conducta y decidir la técnica quirúrgica a emplear, en una patología potencialmente curable.

En la presente comunicación, damos a conocer dos pacientes que fueron estudiadas en nuestros Servicios, las cuales, luego de confirmarse niveles de insulinemia inapropiadamente elevados durante episodios de hipoglicemia espontáneos y con estudio imagenológico no invasivo negativo, fueron sometidas a un estudio angiográfico y de estimulación intraarterial selectiva con calcio (EIS), con medición de insulinemias en venas suprahepáticas, el que permite localizar con certeza el tumor y realizar una cirugía curativa.

Para esto, se catetenizó vía femoral arteria y vena con catéter cobra o multipropósito $6 \mathrm{~F}$ con guías hidrofilicas para arteria y situando el catéter venoso (catéter de Swan Ganz) en vena suprahepática para recolección de muestras de insulinemias. Con el catéter arterial se estimula selectivamente, con gluconato de calcio $(0,025 \mathrm{mEq}$ de $\mathrm{Ca} / \mathrm{kg}$ de peso), en las arterias que imigan la cabeza pancreática (gastroduodenal y mesentérica supenior) y el cuerpo y cola (arteria esplénica) del páncreas y midiendo valores de insulinemia basales y a los 30, 60 y 120 s posteriores a la administración de calcio. La insulinemia fue medida por RIA (Webster, Texas, USA) que tiene una reactividad cruzada de $27,5 \%$ con proinsulina. La sensibilidad del ensayo es $0,8 \mu \mathrm{UI} / \mathrm{mL}$, con coeficiente de variación intraensayo de 3,8\% e interensayo de 4,7\%.

Caso clínico 1. Paciente sexo femenino de 34 años de edad, sin antecedentes mórbidos de importancia, que a principios del año 2001 comenzó con cuadro de astenia, somnolencia y aumento del apetito, agregándose episodios de mareos, sudoración y temblor fino de extremidades. La sintomatología evolucionó en forma progresiva, presentando posteriormente períodos de pérdida transitoria de conciencia y alteraciones conductuales. Inicialmente se diagnosticó trastorno tensional y depresión reactiva, iniciándose tratamiento con psicofármacos. El electroencefalograma resultó normal. Luego fue evaluada por neurólogo en centro privado, planteándose el diagnóstico de epilepsia, por lo que se inició tratamiento anticonvulsivante, pese a lo cual el cuadro descrito persistió. Luego de múltiples consultas en diferentes centros asistenciales, fue hospitalizada en marzo de 2002 en la Asistencia Pública y derivada al Hospital Clínico San Borja Arriarán para estudio. Al examen físico destaca obesidad (IMC de $35 \mathrm{~kg} / \mathrm{m}^{2}$ ) y acantosis nigricans junto a una glicemia de $34 \mathrm{mg} / \mathrm{dl}$, planteándose como primera posibilidad diagnóstica un insulinoma. Se realizó test de ayuno (glicemia en ayunas con insulinemia simultánea). La glicemia fue de $31 \mathrm{mg} / \mathrm{dL}$ y la insulinemia de $31,8 \mu \mathrm{U} / \mathrm{mL}$, compatible bioquímicamente con un insulinoma.

Por lo anterior, se realizó estudio de localización con TAC abdominal y ultrasonografía endoscópica pancreática los cuales resultaron negativos. Se realizó angiografía pancreática la que muestra una imagen vascularizada de 2 x 2 centímetros en relación a la cabeza del páncreas. Con este resultado y para precisar la funcionalidad de la lesión encontrada se sometió a EIS con calcio de acuerdo al protocolo descrito por Doppman ${ }^{6}$.

Los resultados (Figura 1) concuerdan con la imagen obtenida en la angiografía y sugieren la presencia de un insulinoma localizado en la cabeza del páncreas. Fue sometida a cirugía, donde se palpó una lesión en cara anterior de la cabeza de aproximadamente 1,5 por 2 centímetros de diámetro, enucleándose el tumor. La biopsia rápida fue compatible con un tumor neuroendocrino del páncreas confirmándose posteriormente el diagnóstico histológico definitivo de insulinoma.

Caso clínico 2. Paciente de sexo femenino de 30 años. Puérpera de un mes, con antecedente de insulinoma operado ocho años antes, manteniéndose en los años siguientes asintomática. Durante el primer trimestre del último embarazo presentó convulsiones por lo que fue tratada con fenitoína, cesando en segundo y tercer trimestre. Se reinician en el puerperio. Ingresó al Servicio de Urgencia del Hospital Padre Hurtado por convulsión e hipoglicemia severa de $19 \mathrm{mg} / \mathrm{dL}$. Mantuvo glicemias en límite bajo a pesar de infusión de solución glucosada al $10 \%$. 
Se realizó test de ayuno corto, a las $4 \mathrm{~h}$ glicemia de $39 \mathrm{mg} / \mathrm{dL}$, con insulinemia de 7,8 $\mu \mathrm{UI} / \mathrm{mL}$. Se realizó ecotomografía y TAC de abdomen, que no logran identificar el tumor pancreático. Se envió para realización de arteriografía con EIS con calcio, cuyo resultado se presenta en Figura 2.

El test demostró una hiperrespuesta en relación con la estimulación de arteria mesentérica superior, sin respuesta en gastroduodenal ni esplénica, compatible con una lesión en el proceso uncinado del páncreas, hallazgo que fue confirmado durante cirugía y estudio histológico.

\section{Discusión}

La localización de insulinomas con métodos no invasivos siempre ha tenido un bajo rendimiento. El TAC convencional, incluida la técnica helicoidal alcanza una sensibilidad cercana al 40\%. La resonancia nuclear magnética ha reportado éxito en la localización de insulinomas en 60\%. La medicina nuclear en este particular caso es poco útil. El octreotide, análogo de somatostatina marcado con In-111, y que une a receptores de ésta, tiene un rendimiento no superior al 50\%, por la escasez de receptores para somatostatina en este tumor? ${ }^{7}$. Más recientemente se han comunicado mejores resulta$\operatorname{dos}^{8,9}$, pero muy por debajo de la EIS con calcio, lo que asociado a su alto costo lo hacen no elegible.

Las células tumorales de un insulinoma tienen una respuesta exagerada a la estimulación con calcio. Sabemos que el calcio intracelular media en forma fisiológica la exocitosis de insulina por la movilización de los gránulos preformados de ésta en la célula beta. En las células tumorales, esta respuesta está expresada por una sobrerrespuesta de las células tumorales al incremento en la concentración de calcio citosólico.

Doppman, en $1991^{10}$, fue el primero en describir la técnica de estimulación intraarterial con calcio para inducir una respuesta de secreción
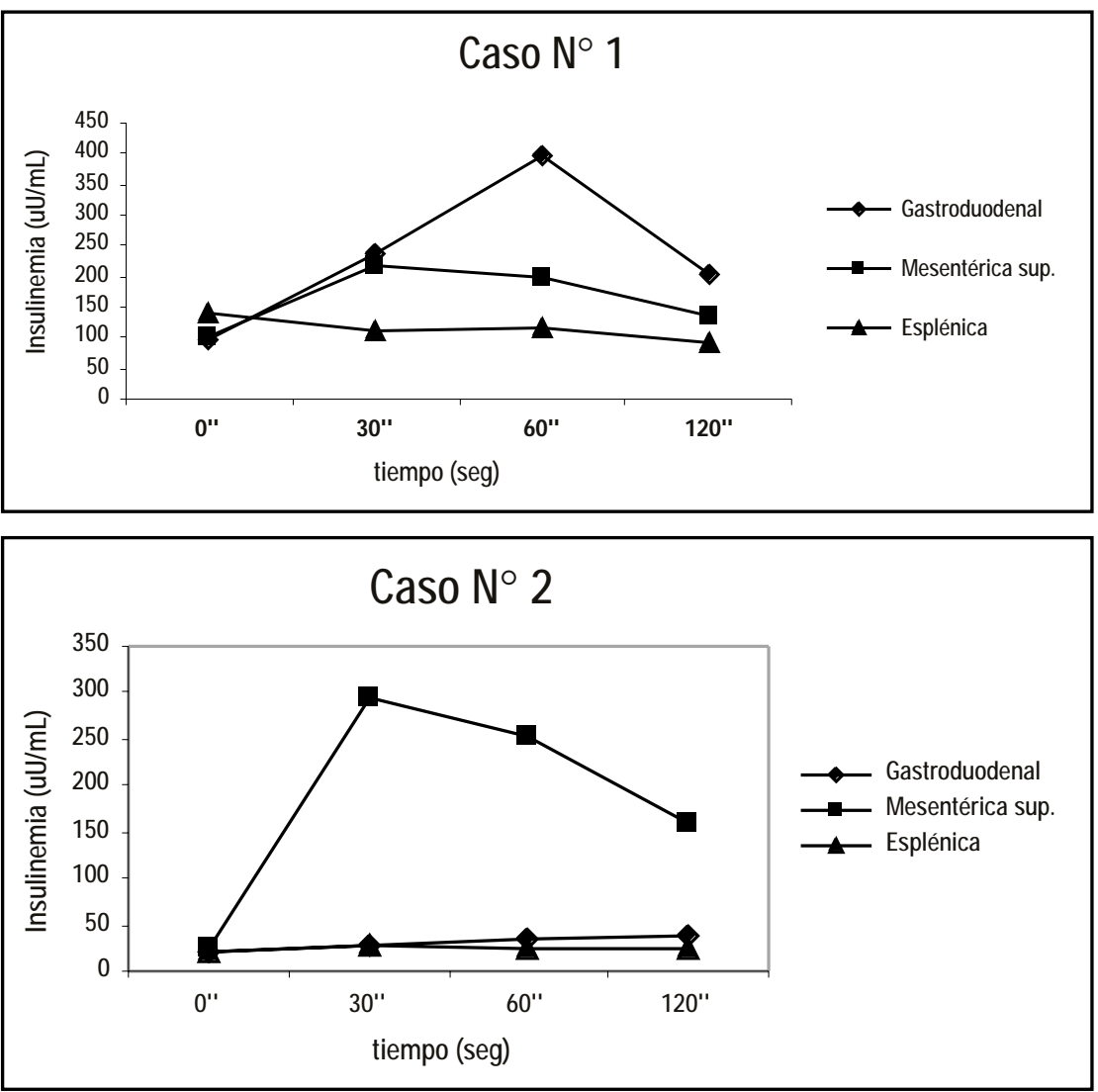

Figura 1. Valores de insulinemia en relación al tiempo, luego de estimulación arterial selectiva con calcio en el caso 1.

FIGURA 2. Valores de insulinemia en relación al tiempo, luego de estimulación arterial selectiva con calcio en el caso 2. 
insulínica lo suficientemente potente para ser detectada en una muestra de sangre venosa temprana, de manera de evaluar el área de circulación pancreática y lograr localizar el tumor. Si al estimular a través de la arteria gastroduodenal o mesentérica superior con una concentración conocida de calcio se eleva la insulinemia más de 2 veces el basal, entonces el tumor debe estar en la cabeza del páncreas o en el cuello, como en el caso de nuestras pacientes. Si al estimular la arteria esplénica se produce el efecto descrito, el tumor lo debemos buscar en el cuerpo y cola pancreáticos. Este estímulo no afecta las células de los islotes normales porque están inhibidas por el tumor. El método tiene una sensibilidad reportada entre el 85 y $100 \%$ y una especificidad mayor de $90 \%$.

Este método ha sido también aplicado para el diagnóstico del fenómeno de hipoglicemia

\section{REFERENCIAS}

1. Service FJ, Mc Mahon MM, O’Brien PC, Balard DJ. Functioning insulinoma incidence, recurrence and long term survival of patients: a 60 years study. Mayo Clin Proc 1991; 66: 711-9.

2. Service FJ. Hypoglycemic disorders. N Engl J Med 1995; 332: 1144-52.

3. Machado MC, DaCunha JE, Jukemura J ET aL. Insulinoma: diagnostic strategies and surgical treatment. A 22 years experience. Hepatogastroenterology 2001; 48: 854-8.

4. Rosch T, Lightdale CJ, Botet JF, Boyce GA, Sivak MV JR, YASUDA K ET AL. Localization of pancreatic endocrine tumors by endoscopic ultrasonography. N Engl J Med 1992; 326: 1721-6.

5. НіRAмото JS. Intraoperative ultrasoundand preoperative localization detects all occult insulinomas. Arch Surgery 2001; 136: 1020.

6. Doppman JL, Chang R, Fraker D, Norton J, AlexanDER R, MuLER DL ET AL. Localization of insulinomas to regions of the pancreas by intra-arterial stimulation with calcium. Ann Int Med 1995; 123: 26973.

7. Meko JB, Doberty GM, Siegel BA, Norton JA. Evaluation of somatostatin receptor scintigraphy for detection neuroendocrine tumors. Surgery 1996; 120: 975-83. hiperinsulinémica en la infancia ${ }^{11}$ y también podría hacer sospechar una hipoglicemia facticia cuando no se produce respuesta frente al estímulo en ninguna de las tres arterias mencionadas ${ }^{12,13}$, aunque también se ha descrito un caso de falso positivo con EIS en hipoglicemia facticia ${ }^{14}$.

La EIS con calcio agrega sólo unos minutos adicionales a la arteriografía, demorando el procedimiento total alrededor de treinta minutos y su relación costo efectividad es alta comparado con otros métodos de imágenes.

En nuestro medio, la realización de este procedimiento no había sido descrita, por lo que nos parece de utilidad presentarlo como un arma de diagnóstico complementaria a los test no invasivos frente al dilema clínico que constituyen los insulinomas ocultos.

8. Schiшaci O, Massa R, Scopinaro F. 111In-pentetreotide scintigraphy in the detection of insulinomas: importance of SPECT imaging. J Nucl Med 2000; 41: 459-62.

9. Chirletti P, Caronna R, Tambrumano G, Mellozzi M, Bonfaciro A, Catalomo C et al. Topographic diagnosis and surgical treatment of insulinoma. Chir Ital 2000; 52: 11-6.

10. Doppman JL, Milier DL, Chang R, Shawker TH, GoRdEN P, NoRTON JA. Insulinomas: localization with selective intraarterial injection of calcium. Radiology 1991; 178: 237-41.

11. Abernethy LJ, Davidson DC, Lamont GL, Shepherd $\mathrm{RM}$, DunNe MJ. Intraarterial calcium stimulation test in the investigation of hyperinsulinaemic hypoglycaemia. Arch Dis Child 1998; 78: 359-63.

12. Palomares R, Zurera L, Gálvez MA, Tofe S, Canis M, BENITO P. Utility of arteriography with selective arterial calcium injection for the diagnosis of insulinoma. Med Clin (Bar) 2002; 119: 568-70.

13. O'Shea D, Rohrer-Therus AW, Lynn JA, Jackson JE, BLoom JR. Localization of insulinomas by selective intraarterial calcium injection. J Clin Endocrinol Metab 1996; 81: 1623-7.

14. Boaz Hirshberg, Skarulis M, Pucino F, Csako G, BREnNAN R, Gorden P. Repaglinide-induced factitious hypoglycemia. J Clin Endocrinol Metab 2001; 86: 475-7.ç 